\title{
IMPORTANCIA DE LA EVALUACIÓN DE LA DOCUMENTACIÓN ONTOLÓGICA, LA VALIDEZ DEL VOCABULARIO Y LA RIGUROSIDAD TAXONÓMICA EN LA REUTILIZACIÓN DE LAS ONTOLOGÍAS. CASO DE EJEMPLO: ONTOLOGÍAS DE BIODIVERSIDAD.
}

\section{IMPORTANCE OF THE ONTOLOGICAL DOCUMENTARY, VOCABULARY VALIDITY AND TAXONOMIC RIGOUR IN ONTOLOGIES REUSE. CASE IN POINT: BIODIVERSITY ONTOLOGIES.}

\author{
Doris Mejia ${ }^{1}$, Antonio Vázquez. ${ }^{2}$, Luis Vilches. ${ }^{3}$ \\ Recibido para publicación: 19 enero de 2013 - Aceptado para publicación: 4 de mayo 2013
}

\section{RESUMEN}

Este artículo destaca para el dominio de la biodiversidad, la importancia de parámetros referentes al contenido de información de las ontologías y que son claves en su reutilización tales como la documentación ontológica, el uso de terminología coherente con el dominio de referencia y la rigurosidad taxonómica con la cual se modelan dichos términos en la ontología. Para un grupo de ontologías dispuestas en la web y asociadas al dominio de la biodiversidad se analizaron los parámetros mencionados. Se definió un corpus del dominio el cual fue comparado con los términos incluidos en cada ontología con el fin de determinar la validez del vocabulario. Para el análisis de documentación ontológica, se estableció el porcentaje de anotaciones en lenguaje natural incluidas en cada ontología a nivel general de la ontología, a nivel de clases, propiedades y axiomas de clase. Para el análisis de la rigurosidad taxonómica de la ontología se identificaron algunos casos de Se encontró que la mayoría de ontologías revisadas se encuentran poco documentas: no documentan el propósito, el nivel de formalidad, alcance, usuarios y usos de la ontología; adicionalmente presentan

\footnotetext{
1 Departamento de Geografía y Medio Ambiente, Universidad de Córdoba, Carrera 6 No. 76-103, Montería, Córdoba, Colombia.

2 Doctor Escuela Técnica Superior de Ingenieros en Topografía, Geodesia y Cartografía Escuela Técnica Superior de Ingenieros en Topografía, Geodesia y Cartografía de la Universidad Politécnica de Madrid (UPM). 28031 Madrid. Autovía de Valencia km 7,5. Madrid, España

3

3 Grupo de Ingeniería Ontológica, Departamento de Inteligencia Artificial, Facultad de Informática - Universidad Politécnica de Madrid, Campus de Montegancedo, sn Boadilla del Monte, 28660, Madrid
} 


\section{Elvis Doris Mejía, documentación Ontológica}

pocas anotaciones en lenguaje natural para clases, propiedades, axiomas e instancias. El vocabulario modelado coincide en bajo porcentaje con el corpus del dominio tomado como referencia. Este análisis permite concluir que en el contexto de desarrollo ontológico es necesario conceder mayor relevancia a los aspectos asociados al contenido de información con el fin de potencializar la reutilización de las ontologías.

Palabras clave: Evaluación ontológica, reutilización de ontologías, ontologías de biodiversidad, anotaciones en lenguaje natural, validez del vocabulario, rigurosidad taxonómica.

\section{ASBTRACT}

This article highlights the importance of ontological documentation as a key in the reuse of ontologies, focusing the biodiversity domain. The information content of the ontologies Biodiversity and Living Element was evaluated, taking into account three parameters: The ontologies documentation (semantic annotations), vocabulary validity and taxonomic riguor. It was found that the revised ontologies do not document the purpose, level of formality, scope, users, and uses of the ontology. These have very few natural language annotations for classes, properties, axioms and instances; there is low coincidence between the modeling vocabulary and the domain corpus. This analysis allows concluding that in the ontological development context is necessary more emphasis on the ontological documentation phase as a key to the reuse of ontologies

Keywords: Ontological evaluation, ontologies reuse, biodiversity ontologies, natural language annotations, validity vocabulary, rigour taxonomic

\section{INTRODUCTION}

Durin the last two decades, the utilization of supercritical carbon dioxide $\left(\mathrm{SC}-\mathrm{CO}_{2}\right)$ as a reaction medium in biocatalysis has been receiving increased attention as potential clean chemical synthesis due to environmentallycompatibility, zero chemical residue in the product, total replacement of organic solvent, high

La biodiversidad es un extraordinario ejemplo de un domino científico que incluye conceptos de diversas áreas y grupos de datos heterogéneos (Gomes, Medeiros 2007), en el cual los datos son provistos por distintos grupos de investigadores y usando diferentes vocabularios (Daltio et al 2008). En otras palabras, el corpus del conocimiento de la biodiversidad toma elementos de múltiples dominios como la Biología, la Ecología, las Ciencias Ambientales y Sociales.
La Informática de la Biodiversidad ha surgido como un conjunto de técnicas informáticas que pueden ampliar los enfoques tradicionales de la Bioinformática mediante la articulación de información a nivel de organismos (Sarkar, 2007). La informática de la biodiversidad aplica tecnologías de la información para la adquisición, almacenamiento, acceso, distribución y análisis de datos concernientes a organismos, poblaciones y taxones biológicos y sus interacciones (Thau et al 2009).

Entre los recursos de la informática que han incursionado en el ámbito de la biodiversidad se encuentran las ontologías. Una ontología es una especificación explicita de una conceptualización. Una conceptualización es una vista abstracta y simplificada del mundo que deseamos representar para algún propósito (Gruber 1993); Las ontologías 


\section{Elvis Doris Mejía, documentación Ontológica}

proporcionan una representación formal de la terminología y conceptos de un dominio científico, utilizándose para aclarar las relaciones entre términos y conceptos (Madin et al 2008).

En el área de las ontologías, para el medio ambiente y la biodiversidad, se han realizado diversos esfuerzos de investigación tales como Web Semántica para la Terminología Ambiental y de la Tierra (SWEET1), Semantic Prototypes in Research Ecoinformatics (SPIRE2), The Science Environment for Ecological Knowledge (SEEK3), Plant Ontology Consortium (PlantOntology4), National Center for Biomedical Ontology, Biodiversity Information Standards (TDWG5), Altern-Net6.

Estos esfuerzos se han orientado al desarrollo de ontologías para los dominios de la biodiversidad y ambiental, las cuales serán integradas en la generación de redes ontológicas que cubran paulatinamente estos dominios. Lo anterior implica llevar a cabo la evaluación de la calidad de estos productos con el fin de garantizar la calidad de los nuevos desarrollos

En el marco de reutilización de ontologías de biodiversidad, se evaluó el vocabulario de las ontologías Biodiversity7, BioEcology8, PhenEcology7, LivingElement7, CRW9, y HumanActivities10 tomando en cuenta tres parámetros: soporte documental de las ontologías, validez del vocabulario y rigurosidad taxonómica.

Se encontró que las ontologías no documentan las especificaciones de requerimientos para su construcción, presentan muy pocas anotaciones en lenguaje natural para clases, propiedades, axiomas e instancias. El

\footnotetext{
${ }^{1}$ http://sweet.jpl.nasa.gov/

$2 \mathrm{http}: / /$ marinemetadata.org/conventions/ontologies-thesauri

http://seek.ecoinformatics.org/Wiki.jsp\%3Fpage=SEEKObservati onOntology.html

${ }^{4}$ http://www.plantontology.org/

$5 \mathrm{http}: / /$ www.tdwg.org/

${ }^{6}$ http://r21854.ovh.net:8085/ontologies/Alternet/

7 http://spire.umbc.edu/ont/

${ }^{8} \mathrm{http}: / /$ sweet.jpl.nasa.gov/2.3

9 http://aims.fao.org/aos/cwr.owl

${ }^{10} \mathrm{http}: / /$ sweet.jpl.nasa.gov/1.1
}

vocabulario modelado coincide en bajo porcentaje con el corpus del dominio y hay algunas ambigüedades taxonómicas difíciles de aclarar, debido principalmente a la falta de documentación y de definiciones formales.

\section{MATERIALES Y METODOS}

Adoptando parte del enfoque para evaluación de ontologías propuesto por Brank et al 2005, Strasunskas y Tomassen 2008, Kehagias et al 2008, Vrande"ci' 2009, referente a la evaluación del léxico para determinar la cohesión entre el dominio y el vocabulario usado en la ontología, se analizó el contenido de información de algunas ontologías asociadas al dominio de la biodiversidad, tales como: Biodiversity, EcologicalConcept, EcologicalModels, LivingElement, CWR (FAO) y HumanActivities. Se analizaron tres parámetros:

Validez del vocabulario: La validez del vocabulario se refiere a los términos significativos en otras fuentes del conocimiento que están incluidos en la ontología. Para evaluar este parámetro se retomó el método usado por Brewster et al. (2004), identificando un conjunto de fuentes bibliográficas que conforman el corpus del dominio; de cada una de estas fuentes se elaboraron listados de términos que fueron comparados con los que constituyen la ontología objeto de evaluación.

Nivel de documentación de las ontologías mencionadas. Las ontologías se documentan mediante la inclusión de anotaciones en lenguaje natural dentro de la ontología. Una ontología concebida como un recurso de conocimiento que permite precisar conceptos de un dominio, implica la existencia de una base conceptual en leguaje natural que soporte el conocimiento formalizado por la ontología. Dicha base conceptual en el marco de la ontología puede constituirse básicamente por dos elementos:

a) Una memoria que describa el contexto general de la ontología (el propósito, el nivel de formalidad, alcance, usuarios, usos de la ontología y demás información útil para 


\section{Elvis Doris Mejía, documentación Ontológica}

orientar su construcción). Por ejemplo en la metodología NeOn para construcción de redes ontológicas propuesta por Suarez y Figueroa et al 2009, la memoria de la ontología se almacena en un documento denominado

"documento de especificación de requerimientos de la ontología ORSD (Ontology Requirements Specification

Document)". Adicionalmente dicha memoria puede ser incluida en la ontología mediante anotaciones en lenguaje natural.

b) Un soporte conceptual de la formalización de la ontología.

La mayoría de lenguajes ontológicos permiten incluir estos dos elementos de documentación dentro de la ontología mediante el uso de anotaciones en lenguaje natural. Las anotaciones se hacen a nivel de contexto general de la ontología y para descripción de clases, instancias, axiomas y relaciones.

La evaluación de la documentación de las ontologías consistió en determinar la existencia de anotaciones en lenguaje natural tanto a nivel de descripción general de la ontología como a nivel de clases, de instancias, de propiedades y de axiomas.

Rigurosidad taxonómica: consiste en determinar la claridad, consistencia y exhaustividad de modelado de los conceptos dentro de la ontología. Se revisó la estructura jerárquica de las ontologías, verificando la completitud 0 exhaustividad de clases y chequeando inconsistencias taxonómicas tales como clases no excluyentes o mal jerarquizadas.

\section{RESULTADOS Y DISCUSIONES}

\subsection{VALIDEZ DEL VOCABULARIO}

El análisis de la validez del vocabulario para un domino como la biodiversidad es complejo dada su extensión y la transversalidad que tiene este con otros como la Biología, la Ecología y las Ciencias Ambientales. En este sentido es difícil definir un número de términos concretos, porque todo dependerá de la escala o nivel de detalle al que se maneje el dominio y de los objetivos que persiga el recurso semántico (vocabulario controlado, tesauro u ontología). De esta manera la metodología utilizada aquí para identificar los términos más usuales en este dominio no pretende determinar listados definitivos, sino orientarse un poco en términos comunes que puedan servir de base de comparación con los utilizados en la ontología objeto de análisis.

Se definió un corpus del dominio integrado por textos y glosarios referentes a vocabulario específico del dominio de la biodiversidad tales como: "Encyclopedia of Biodiversity" (Levin, 2001), el glosario de biodiversidad del World Resources Institute ${ }^{1}$, el glosario de biodiversidad del mecanismo de Clearing House de Comunidad Europea de Biodiversidad (European Community Biodiversity Clearing House Mechanism ${ }^{2}$ ), y el glosario de Biodiversidad de la Convención de Diversidad Biológica (Convention on Biological Diversity $^{3}$ ). Para el análisis se determinó el número total de términos de la ontología presentes en cada uno de los listados proporcionados por el corpus mencionado.

Tabla 1. Porcentaje de términos de la ontología

"Biodiversity" incluidos en el corpus de dominio.

\begin{tabular}{|l|l|l|}
\hline Recurso & $\begin{array}{l}\text { Número total } \\
\text { de términos } \\
\text { que } \\
\text { constituyen el } \\
\text { recurso }\end{array}$ & $\begin{array}{l}\text { Porcentaje de } \\
\text { términos de la } \\
\text { ontología } \\
\text { encontrados en } \\
\text { el recurso }\end{array}$ \\
\hline $\begin{array}{l}\text { Encyclopedia of } \\
\text { Biodiversity }\end{array}$ & 1930 & $17 \%$ \\
\hline $\begin{array}{l}\text { Biodiversity glossary } \\
\text { of World Resources } \\
\text { Institute }\end{array}$ & 107 & 4.6 \\
\hline $\begin{array}{l}\text { Glossary of } \\
\text { biodiversity of } \\
\text { European } \\
\text { Community } \\
\text { Biodiversity Clearing } \\
\text { House Mechanism }\end{array}$ & 574 & 12,2 \\
\hline $\begin{array}{l}\text { Glossary of } \\
\text { biodiversity of } \\
\text { Convention on } \\
\text { Biological Diversity }\end{array}$ & 1388 & $6 \%$ \\
\hline
\end{tabular}

\footnotetext{
1 http://www.wri.org/publication/content/8183

$2 \mathrm{http}: / /$ biodiversity-chm.eea.europa.eu/nyglossary_terms

${ }^{3} \mathrm{http}: / /$ www.cbd.int/doc/cbd-voc.aspx
} 


\section{Elvis Doris Mejía, documentación Ontológica}

La tabla 1 presenta los resultados de la comparación. En ésta se incluye el nombre de cada producto del corpus definido, el número de términos totales que forman el producto y el porcentaje de términos de cada una de las ontologías que fue encontrado en dicho corpus. De acuerdo a la tabla, ninguno de los productos semánticos tomados como referencia incluye más del $17 \%$ de los términos incluidos en las ontologías evaluadas.

\begin{tabular}{|c|c|c|c|c|c|c|c|}
\hline Nombre del & \# térr & inos d & finidos & en el $p$ & oductc & & \\
\hline & & 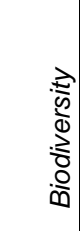 & 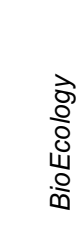 & $\begin{array}{l}\text { ठे } \\
\text { ठ } \\
\text { 仓 } \\
\frac{1}{\delta} \\
\frac{1}{2}\end{array}$ & $\begin{array}{l}\frac{0}{0} \\
\frac{1}{1} \\
\frac{\pi}{3} \\
\frac{0}{0}\end{array}$ & 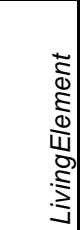 & 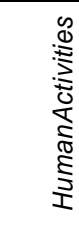 \\
\hline $\begin{array}{l}\text { Encyclopedia } \\
\text { of Biodiversity }\end{array}$ & $\begin{array}{l}193 \\
0\end{array}$ & $\begin{array}{l}17 \\
\%\end{array}$ & $\begin{array}{l}10 \\
\%\end{array}$ & $4 \%$ & $\begin{array}{l}10 \\
\%\end{array}$ & $9 \%$ & $4 \%$ \\
\hline $\begin{array}{l}\text { GLOSARIO } \\
\text { DE } \\
\text { BIODIVERSID } \\
\text { AD DEL } \\
\text { WORD } \\
\text { RESOURCE } \\
\text { INSTITUTE }\end{array}$ & 107 & 4.6 & $\begin{array}{l}3.4 \\
\%\end{array}$ & $\begin{array}{l}1.8 \\
\%\end{array}$ & $\begin{array}{l}6.5 \\
\%\end{array}$ & $\begin{array}{l}4.6 \\
\%\end{array}$ & $\begin{array}{l}2.8 \\
\%\end{array}$ \\
\hline $\begin{array}{l}\text { GLOSARIO } \\
\text { DE } \\
\text { BIODIVERSID } \\
\text { AD DEL } \\
\text { European } \\
\text { Community } \\
\text { Biodiversity } \\
\text { Clearing } \\
\text { House } \\
\text { Mechanism }\end{array}$ & 574 & $\begin{array}{l}12, \\
2\end{array}$ & $6 \%$ & $\begin{array}{l}3.5 \\
\%\end{array}$ & $\begin{array}{l}5,2 \\
\%\end{array}$ & $6 \%$ & $2 \%$ \\
\hline $\begin{array}{l}\text { Vocabulario } \\
\text { controlado de } \\
\text { la Convención } \\
\text { de Diversidad } \\
\text { Biológica }\end{array}$ & $\begin{array}{l}138 \\
8\end{array}$ & $6 \%$ & 3.6 & 0,4 & 6 & 1.8 & 0.9 \\
\hline
\end{tabular}

Tabla 1. Términos definidos del producto

Más que referirse al bajo porcentaje de coincidencia entre el corpus del dominio definido y los términos incluidos en la ontología se resalta la importancia de conocer el contexto general de la ontología, ya que este justificará el conjunto de términos aglutinados en una ontología como útiles en una aplicación concreta. De esta forma se evidencia nuevamente la necesidad de documentación a nivel general de la ontología (propósito, el nivel de formalidad, alcance, usuarios, usos de la ontología).

\subsection{Documentación ontológica}

En la tabla $x x$ se presenta una síntesis de algunas métricas para las ontologías evaluadas.

\begin{tabular}{|c|c|c|c|c|c|c|c|c|c|}
\hline & $\begin{array}{l}8 \\
\frac{\pi}{1} \\
\frac{1}{5} \\
0\end{array}$ & 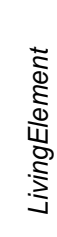 & 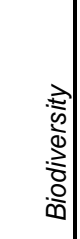 & 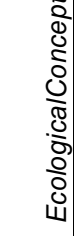 & 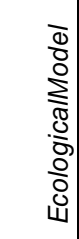 & $\begin{array}{l}0 \\
0 \\
0\end{array}$ & & & \\
\hline $\begin{array}{l}\text { Númer } \\
0 \text { de } \\
\text { clases }\end{array}$ & $\begin{array}{l}103 \\
6\end{array}$ & $\begin{array}{l}8 \\
6\end{array}$ & $\begin{array}{l}16 \\
6\end{array}$ & $\begin{array}{l}16 \\
3\end{array}$ & $\begin{array}{l}40 \\
9\end{array}$ & 159 & & & \\
\hline \multicolumn{3}{|c|}{ Propiedades } & \multicolumn{2}{|l|}{77} & 0 & 32 & 32 & $\begin{array}{l}14 \\
3\end{array}$ & 6 \\
\hline \multicolumn{3}{|c|}{ Atributos } & & 0 & 1 & 1 & 38 & 0 \\
\hline \multicolumn{3}{|c|}{ Instancias } & \multicolumn{2}{|l|}{549} & 0 & $\begin{array}{l}10 \\
8\end{array}$ & 75 & $\begin{array}{l}11 \\
5\end{array}$ & 0 \\
\hline \multicolumn{3}{|c|}{ Axiomas de clase } & \multicolumn{2}{|l|}{1069} & 88 & $\begin{array}{l}18 \\
8\end{array}$ & $\begin{array}{l}18 \\
8\end{array}$ & $\begin{array}{l}56 \\
1\end{array}$ & $\begin{array}{l}15 \\
2\end{array}$ \\
\hline \multicolumn{3}{|c|}{$\begin{array}{l}\text { Porcentaje de } \\
\text { anotaciones a nivel } \\
\text { de clase }\end{array}$} & \multicolumn{2}{|l|}{0} & 0 & 55 & 42 & 42 & 0 \\
\hline
\end{tabular}

Tabla 2. Ontologías evaluadas

Ninguna de las ontologías listadas en la tabla xx presenta anotaciones a nivel de ontología, lo cual no permite documentarse acerca del propósito, nivel de formalidad, alcance, usuarios y usos de la ontología. Tampoco se presentan anotaciones en lenguaje natural para propiedades, atributos y axiomas de clase. A nivel de clases, las anotaciones en lenguaje natural también son escasas, en ninguna de las ontologías se supera el $55 \%$. A continuación se presentan algunos ejemplos tomados de las ontologías Biodiversity y Living Element, los cuales sustenta la importancia que tiene el soporte documental (anotaciones en lenguaje natural) en el entendimiento y selección de ontologías para su reutilización:

Algunos conceptos son totalmente incomprensibles, porque el nombre asignado a la clase proviene de mezclas de palabras como por ejemplo la clase

ComputableTraitParametArray presente en la ontología Biodiversity, la cual no tiene anotaciones y tampoco axiomas que la definan formalmente. Al respecto las anotaciones a nivel de clase para este tipo de términos serían 


\section{Elvis Doris Mejía, documentación Ontológica}

útiles para un mayor entendimiento de los mismos.

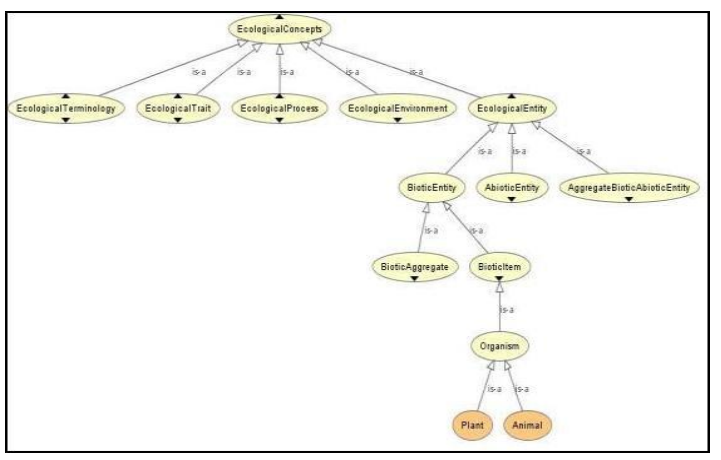

Figura 1. Fragmento de la ontología "Biodiversity". Se puede observar la línea jerárquica que recorre las clases BioticEntity, Bioticltem, Organism, Plant y Animal

Biodiversity y Living Element comparten conceptos universales como Animal y Plant; sin embargo, estos son modelados en forma diferentes para cada una de estas ontologías. En la ontología LivingElement se puede observar la línea jerárquica que recorre las clases BioticEntity, Bioticltem, Organism, Plant y Animal (figura 1). De estos términos, solamente se encuentran definidos en lenguaje natural BioticEntity (entidad viva, posiblemente compuesta por múltiples sub-entidades) y Bioticltem (entidad viva individual definida como la base en la entidad biótica agregada a la cual pertenece el individuo)

En Biodiversity los conceptos Animal y Plant han sido descritos formalmente con

condiciones necesarias y suficientes [Organism and taxonomiclD only

AnimalTaxonomicldentifier], es decir un animal o una planta debe cumplir la condición de ser un organismo y su identificación taxonómica solamente puede corresponder a la clase AnimalTaxonomic o PlantTaxonomic. Además esta definición formal se complementa con las restricciones asociadas a su superclase, que se refieren a que un ser vivo individual siempre es parte de una agregación de seres vivos (Organism: [partOf only

AggregateBioticAbioticEntity]), solo puede pertenecer a algo ([partOf only SetOfOrganisms], [partOf only
BioticAggregate), [partOf exactly 1 Thing]), posee rasgos que lo individualizan y lo hacen pertenecer a una etapa de la vida [hasTraits only (BioticltemTrait or LifeStageTrait)]).

De lo anterior surge un interrogante: ¿Por qué dos conceptos tan universales como Animal y Plant son definidos y jerarquizados de manera diferente en estas ontologías? Una vez más este interrogante podría ser resuelto si las anotaciones a nivel de ontología aportaran información acerca de su contexto general.

En la ontología Living Element las entidades Animal y Plant son subclases de la clase LivingThing (figura 2). Estas no presentan axiomas que las definan formalmente $y$ tampoco anotaciones a nivel de clase que las definan en lenguaje natural. Sin embargo, de acuerdo a las subclases asociadas a Animal (Insect, mamal, fish, etc.) se podría presumir que se trata de una clase abstracta que alberga una clasificación artificial para los animales. En el marco ontológico las presunciones son contrarias a su fundamento: una ontología "es una especificación explicita de una conceptualización"; de esta forma, la construcción de ontologías debe incluir los elementos necesarios que eliminen cualquier indicio de ambigüedad y por tanto la necesidad de presunción.

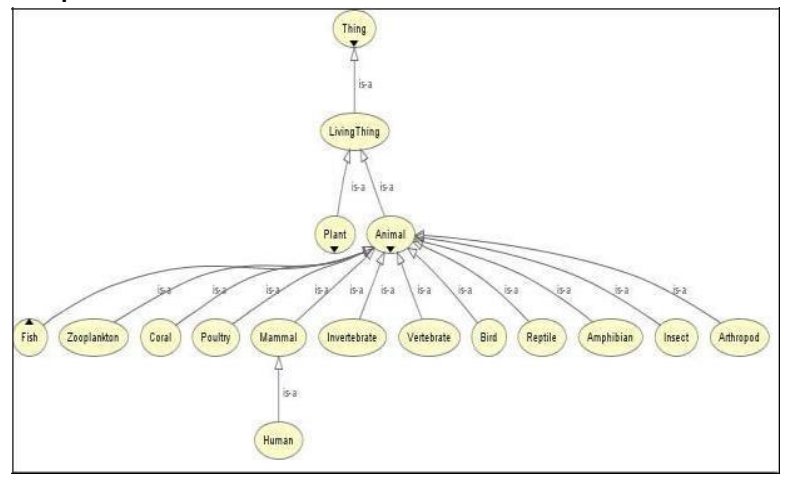

Figura 2. Vista gráfica del primer y segundo nivel de términos de la ontología "Living Element".

Otro ejemplo de la necesidad de anotaciones y definiciones formales se evidencia al intentar entender la jerarquización de la clase Plant en la ontología Living Element (figura 3); entre las subclases asociadas a la Plant se encuentra Crop, la cual no presenta anotaciones y tampoco axiomas que la definan 


\section{Elvis Doris Mejía, documentación Ontológica}

formalmente. En la búsqueda de fuentes de información para entender el concepto Crop se encontró que es un término homónimo, con las siguientes definiciones:

a) Conjunto biológico de organismos (células, tejidos, etc.) parecidos entre sí y cuyo crecimiento y reproducción se observan en relación con condiciones de luz, temperatura, humedad, gases, presiones, etc. y que se utilizan como medio de diagnóstico (Barioglio, 2006)

b) Cultivo agrícola o forestal (comunidad vegetal desarrollada mediante prácticas culturales adecuadas, las cuales son utilizadas para beneficio del hombre) (Barioglio 2006)

¿A cuál de las dos definiciones hace referencia el término Crop en la ontología Living Element? Se podría suponer que Crop en esta ontología se está refiriendo a cultivos agrícolas, sin embargo en este caso algunas subclases de Plant no serían excluyentes, tal es el caso de Crop, FloweringPlant y Vegetación.

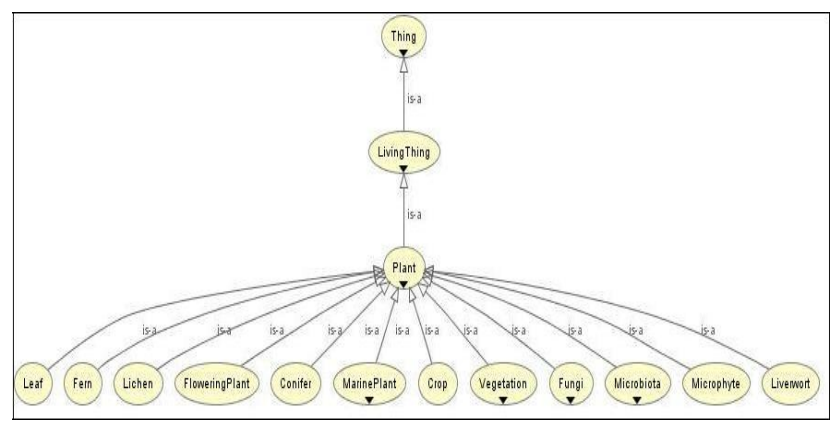

Figura 3. Fragmento de la ontología "Living Element". Clase Plant con algunas de las subclases asociadas a ésta.

En el caso de la reutilización de estos productos, si un experto de dominio tiene que elegir una de las dos ontologías descritas anteriormente, es posible que no cuente con los suficientes elementos para determinar cuál se ajusta más a sus necesidades. La potencialidad de reutilización de la ontología sería mayor, si el experto de dominio dispusiera de una síntesis de la memoria de la ontología (los objetivos de construcción de la ontología, el alcance, el tipo de usuarios hacia los que se orienta y los posibles usos que puede tener) o incluso si esta se encontrará almacenada en las anotaciones dentro de la ontología. El entendimiento del contexto de construcción de la ontología es fundamental para comprender el vocabulario, las propiedades y relaciones incluidas en esta.

\subsection{Rigurosidad taxonómica}

La evaluación de la rigurosidad taxonómica considera el chequeo de inconsistencias, completitud y redundancia (Gómez et al 2004). Para evaluar inconsistencias y completitud se utilizó el editor de ontologías Protégé 4. A continuación se ilustran algunos casos específicos de este tipo de errores en las ontologías objeto de análisis:

La falta de completitud taxonómica se hace evidente en la ontología Biodiversity para el término Ecologicallnteraction (figura 4). En las subclases del término InterSpecificInteraction están ausentes categorías como: predación, amensalismo y comensalismo (Morin 1999)

En la ontología Living Element la taxonomía propuesta para la clase Animal es compleja ya que las subclases planteadas para ésta no tienen una característica común que justifique su existencia. Así, ha sido incluida una subclase llamada Mamal, sin incluir dentro de este nivel las clases suplementarias (clases hermanas) de Mammal y que constituirían la taxonomía completa de la clase Animal. Esto implica una falta de exhaustividad entre clases; Adicionalmente algunas de estas subclases no son excluyentes, es el caso de Bird y Poultry

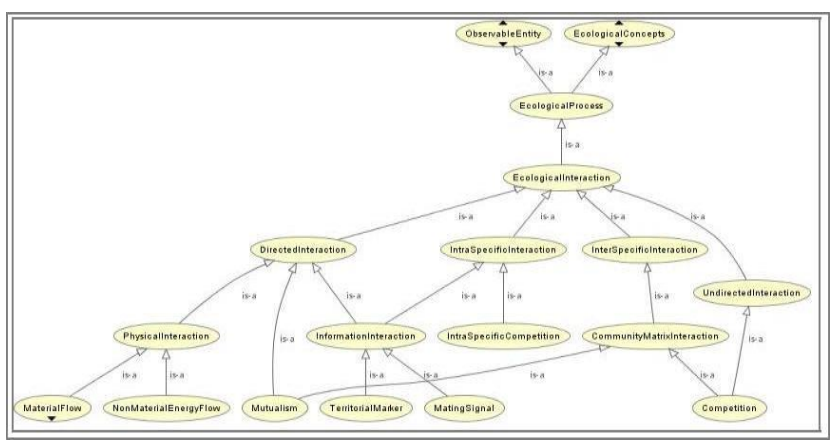




\section{Elvis Doris Mejía, documentación Ontológica}

Figura 4. Esquema gráfico de la taxonomía de clases del concepto Ecologicallnteraction de la ontología "Biodiversity".

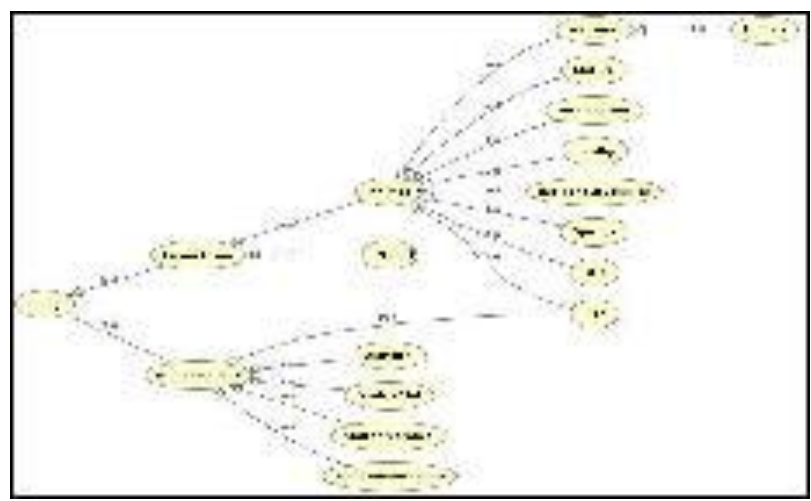

Figura 5. Esquema gráfico de la taxonomía del concepto LivingThig en la ontología "Living Element"

Una inconsistencia taxonómica se ejemplariza con los conceptos LivingThing y MarineAnimal, estructurados como clases del mismo nivel jerárquico y sin definiciones formales que las articule; así podría inferirse que MarineAnimal no es un ser vivo (figura 5). Además, hay algunas subclases de la clase Animal como por ejemplo Fish, Mollusk y Sponge que deberían estar relacionadas con la clase MarineAnimal; sin embargo la única que presenta esta relación es Fish, de tal manera que se excluye a una parte de los moluscos y las esponjas que también se encuentran en ambientes marinos. Además las subclases de MarineAnimal no son mutuamente excluyentes ya que Jellyfish está incluida dentro de los animales marinos invertebrados

(Marinelnvertebrate). Este tipo de inconsistencias pueden estar dadas por el uso de definiciones incompletas de los conceptos, como por ejemplo falta de axiomas que determinen disyunción entre clases.

Por otro lado la definición formal de grandes grupos como plantas y animales en una ontología de alto nivel implicaría definir niveles de detalle 0 estructuras taxonómicas especiales dada la dificultad real de establecer diferencias que abarquen todos los animales 0 todas las plantas, ya que las grandes diferencias bien sea a niveles micro o macroscópico no los contienen en su totalidad, como lo explican Roger y otros (1996) y Yablonski (2005), de la misma forma la definición formal de grupos de microorganismos implicaría incluir conceptos como "Procariotas y Eucariotas" (Alcamo, 2004).

La aparente ambigüedad o presunción de errores en la taxonomía de estos conceptos dentro de la ontología podría disminuir o ser aclarada si adicionalmente se cuenta con mayor número de anotaciones a nivel de clases y de axiomas que completen las definiciones formales de los conceptos.

\section{CONCLUSIONS}

En este artículo se analizaron tres parámetros importantes para el entendimiento y reusabilidad de las ontologías: validez del vocabulario, documentación ontológica y rigurosidad taxonómica, encontrándose que tanto la validez del vocabulario como de la rigurosidad taxonómica son parcialmente dependientes de la adecuada documentación de la ontología.

Dada la estrecha relación de la biodiversidad con otras áreas de conocimiento como la biología, la ecología, la geografía entre otras, se encontró que para determinar la validez del vocabulario en el contexto de ontologías para el dominio de la biodiversidad es fundamental que la ontología se encuentre documentada en relación al contexto general a partir del cual fue construida. Esto puede contribuir a explicar la presencia 0 ausencia de términos en la ontología.

El análisis e interpretación del contenido de información de las ontologías evaluadas como una actividad necesaria durante el proceso de selección de ontologías para su reutilización fue una tarea compleja, dado que las ontologías no presentaron anotaciones a nivel general (los objetivos de construcción de la ontología, el alcance, el tipo de usuarios hacia los que se orienta y los posibles usos que puede tener), anotaciones para instancias, 


\section{Elvis Doris Mejía, documentación Ontológica}

propiedades y axiomas. De igual forma, el porcentaje de anotaciones a nivel de clase fue muy bajo y la mayoría de clases no presentan definiciones formales 0 los axiomas simplemente determinan jerarquías de términos. Lo anterior pone de manifiesto la estrecha relación que existe entre la documentación completa de una ontología y su potencialidad de reutilización.

En cuanto al análisis de la rigurosidad taxonómica, se ilustraron algunos ejemplos de falta de exhaustividad 0 completitud e inconsistencias taxonómicas presentadas en las ontologías Biodiversity y Living Element, las cuales son atribuidas a jerarquizaciones taxonómicas incorrectas y a la falta de definiciones formales que permitan establecer las relaciones entre clases. Se hace evidente la necesidad de documentación a nivel de contexto general de la ontología y a nivel de clases, con el fin de contar con argumentos concretos para comprender el modelado de los conceptos y así determinar inconsistencias, completitud y redundancia.

En el proceso de construcción de ontologías, su documentación posiblemente no cobre gran importancia para los desarrolladores porque éstas no hacen parte de las construcciones lógicas, que permiten alcanzar productos ontológicos con alto nivel de formalidad. Sin embargo el experimento aquí descrito, hace evidente su importancia. Las anotaciones dentro de la ontología como una forma de documentación permiten incluir descripciones en lenguaje natural para cada uno de los diferentes niveles de construcción de la ontología (descripción general de la ontología, descripción de clases, de instancias, propiedades y axiomas), cumpliendo doble función: por un lado orientan al experto en el domino para el entendimiento del lenguaje formal y por otro, los desarrolladores de ontologías las podrían utilizar como base para la construcción de nuevos axiomas o la modificación de los existentes.

Más allá de la decisión específica de reutilizar estas ontologías, el resultado importante es la evidente necesidad de tener en cuenta la importancia de un buen nivel de documentación de la ontología a desarrollar.
De un buen nivel de documentación depende la posibilidad de detectar el grado de pertinencia del vocabulario utilizado en la ontología de acuerdo a un campo de aplicación específico. Adicionalmente, una documentación completa permite precisar en relación a posibles inconsistencias en la definición formal de la terminología o la necesidad de usar nuevos axiomas que complementen dichas definiciones.

Es importante no perder de vista la trascendencia de cada uno de los elementos que actúan en el desarrollo de las ontologías: el conocimiento, el componente técnico y el componente humano. Es posible que también en el ámbito ontológico la velocidad en los avances tecnológicos esté ganando la carrera a los expertos del dominio, quienes son los responsables de posicionar el dominio de conocimiento como el elemento clave para que estos productos puedan cumplir un papel válido apoyando la solución de problemáticas reales.

\section{REFERENCES}

[1]. Y. Ikushima, Adv. Coll. Int. Sci. 71/72 (1997) 259.

[2]. J. Mesiano, E. J. Beckman, A. J. Russell, Supercritical Biocatalysis, Chem. Rev. 99 (1999) 623.

[3]. Alcamo, I.E., 2004. Fundamentals of Microbiology: A Comprehensive Review. 7 edn.

[4]. Avraham, S., Tung, C.W., Ilic, K., Jaiswal, P., Kellogg, E.A., Mccouch, S., Pujar, A., Reiser, L., Rhee, S.Y. and Sachs, M.M., 2008. The Plant Ontology Database: a community resource for plant structure and developmental stages controlled vocabulary and annotations. Nucleic acids research, 36 (Database issue), D449.

[5]. Barioglio, C.F., 2006. Diccionario de las Ciencias Agropecuarias. 1 edn. Ediciones Encuentro. 
[6]. Black, J.G. and LewiS, L.M., 2005. Microbiology: principles and explorations. Wiley.

[7]. Bontas, E.P., Mochol, M. and Tolksdorf, R., 2005. Case studies on ontology reuse, Proc. of the 5th International Conference on Knowledge Management, 2005, Citeseer.

[8]. Brank, J., Grobelnik, M. and Mladenic, D., 2005. A survey of ontology evaluation techniques, Proceedings of the Conference on Data Mining and Data Warehouses (SiKDD 2005), 2005, Citeseer.

[9]. Brewster, C., Alani, H., Dasmahapatra, S. and Wilks, Y., 2004. Data driven ontology evaluation, Proceedings of LREC, 2004, Citeseer.

[10]. Burton-Jones, A., Storey, V.C., Sugumaran, V. and Ahluwalia, P., 2005. A semiotic metrics suite for assessing the quality of ontologies. Data \& Knowledge Engineering, 55(1), 84-102.

[11]. Daltio, J., Medeiros, C.B. and Lewinsohn, T.M., 2008. A framework to process complex biodiversity queries, Proceedings of the 2008 ACM symposium on Applied computing, 2008, ACM New York, NY, USA pp2293-2297.

[12]. Downey, L. and Pennington, D., 2009. Bridging the gap between technology and science with examples from ecology and biodiversity. Biodiversity Informatics, 6(1).

[13]. Fernández, M., Cantador, I. and Castells, P., 2006. CORE: A tool for collaborative ontology reuse and evaluation, Proceedings of the 4th Int. Workshop on Evaluation of Ontologies for the Web (EON'06), at the 15th Int. World Wide Web Conference (WWW'06). Edinburgh, UK, 2006, Citeseer.

[14]. Gomes JR, L.C. and Medeiros, C.B., 2007. Ecologically-aware queries for biodiversity research, Proceedings of
Geolnfo-Brazilian

Geoinformatics

Symposium, INPE-SBC, 2007, Citeseer.

[15]. Gómez-Pérez, A., 1995. Some ideas and examples to evaluate ontologies, caia, 1995, Published by the IEEE Computer Society pp299.

[16]. Gomez-Perez, A., Fernández-López, M. and Corcho, O., 2004. Ontological Engineering: with examples from the areas of Knowledge Management, eCommerce and the Semantic Web. Springer Verlag.

[17]. Gómez-Pérez, A., Fernández-López, M. and CorchO, O., 2004. Ontological Engineering: with examples from the areas of Knowledge Management, eCommerce and the Semantic Web. Springer verlag.

[18]. Gruber, T.R., 1995. Toward principles for the design of ontologies used for knowledge sharing. International Journal of Human Computer Studies, 43(5), 907928.

[19]. Gruber, T.R., 1993. A translation approach to portable ontology specifications. Knowledge Acquisition, 5(2), 199-220.

[20]. Guarino, N. and Welty, C., 2002. Evaluating ontological decisions with OntoClean. Communications of the ACM, 45(2), 65.

[21]. Guarino, N. \& Welty, C.A. 2009, "An overview of OntoClean", Handbook on ontologies, , pp. 201-220.

[22]. Henderson, M., Khan, I. and Hunter, J., 2007. Semantic WildNet: An Ontologybased Biogeographical System.

[23]. HollandeR, A. and Wang, D., 2006. Using the Semantic Web to Support Ecoinformatics, Proceedings of the AAAI Fall Symposium on the Semantic Web for Collaborative Knowledge Acquisition, 2006. 